\title{
Verschiedene Faktoren berücksichtigen
}

\author{
WISSENSCHAFT ERKLÄRT: UNI- UND MULTIVARIATE AUSWERTUNGEN Wollen Forscher \\ herausfinden, warum bestimmte Erkrankungen entstehen, müssen sie die Daten der \\ betroffenen Patienten analysieren. In diesem Zusammenhang spricht man auch von \\ uni- oder multivariaten Analysen. Lesen Sie, worin sich beide unterscheiden und welche \\ Rolle „abhängige“ und „unabhängige“ Variablen in diesem Zusammenhang spielen.
}

n einer Studie, die in einer der letzten Ausgaben der renommierten Fachzeitschrift „Stroke“ veröffentlicht wurde, stellen kanadische Forscher bei Patienten nach Schlaganfall einen Zusammenhang zwischen der Lokalisation der Hirnverletzung und einer Gangasymmetrie fest [1]. Das Ziel der Studie war es, herauszufinden, worin die Ursache für eine schlaganfallbedingte Ganganomalie liegen könnte. An der Untersuchung nahmen 37 betroffene Patienten teil. Zunächst hatten die Wissenschaftler die teilnehmenden Probanden anhand ihres Gangbildes in zwei Gruppen aufgeteilt: Die Patienten mit symmetrischem Gang bildeten die eine, die mit einem asymmetrischen Gang die andere Gruppe. Danach beurteilten die Forscher die Computertomografieaufnahmen, die sie von den Gehirnen der Probanden gemacht hatten. Sie stellten fest, dass bei den Patienten mit asymmetrischem Gang die Hirnschädigung überwiegend im posterolateralen Putamen lag. Die Wissenschaftler zogen aus dieser Entdeckung den Schluss, dass die Lokalisation der Schädigungen für die Gangasymmetrie der Patienten verantwortlich ist.

Univariate Analyse birgt Risiken > Abgesehen davon, dass wahrscheinlich Schädigungen in vielen Hirnbereichen zu motorischen Asymmetrien führen können, haben die Forscher in dieser Studie noch andere Faktoren nur unzureichend berücksichtigt: die prognostischen Werte der Studienteilnehmer. Bei deren Betrachtung fällt auf, dass sich die Gruppen nicht nur hinsichtlich ihres Gangbildes und der Lokalisation der Schädigung unterscheiden, sondern dass auch noch weitere
Faktoren als Erklärung der Gangasymmetrie in Betracht kommen - beispielsweise die Größe der Hirnverletzung.

Wenn Wissenschaftler zur Erklärung eines Parameters, in diesem Fall der Gangasymmetrie, nur einen einzelnen Faktor überprüfen so wie hier die Computertomografieaufnahmen -, spricht man von einer univariaten Analyse. Allerdings bergen Zusammenhänge, die auf einer solchen Basis entdeckt werden, ein hohes Risiko, fehlerhaft zu sein. Es könnte sein, dass dabei erkannte Häufungen, beispielsweise die Putamenschädigung, zufällig sind. Solche Auswertungsfehler können zu einer Verzerrung der Resultate, im schlechtesten Fall sogar zu einer falschen Aussage führen.

Zuverlässigere Ergebnisse durch multivariate Analyse > Um die Gründe für bestimmte Symptome oder Erkrankungen mit einer höheren Sicherheit herausfinden zu können, ist eine sogenannte multivariate Analyse sinnvoller. Bei der multivariaten Analyse überprüfen Forscher einfach mehrere Faktoren auf ihren Zusammenhang mit dem zu erklärenden Parameter ( $\odot$ „Schlaganfall - Beinkraft ausschlaggebend für Aktivitätsniveau“, S. 18).

Um die Frage zu beantworten, welches die Gründe für eine Gangasymmetrie nach Schlaganfall sind, müsste eine sinnvolle Analyse Folgendes beinhalten: Der zu erklärende Parameter, auch „abhängige“ Variable genannt, ist die Gangasymmetrie. Zu den Parametern, welche diese abhängige Variable erklären könnten, gehören neben dem Ort der Schädigung beispielsweise auch demografische Daten wie Alter, Geschlecht, Krankheitsdauer und sozialer Status der Patienten. Auch verschie- dene Körperfunktionen und -strukturen, zum Beispiel die Infarktgröße, der Lähmungsgrad sowie Sensibilitäts- und neuropsychologische Störungen, können nach einem Schlaganfall Auswirkungen auf das Gangbild haben und sollten daher ebenfalls beachtet werden. Diese erklärenden Daten nennt man „unabhängige“ Variablen.

In unserem Beispiel hätte man durch eine multivariate Analyse noch erkennen können, dass die Patienten mit asymmetrischem Gangbild auch in ihren motorischen Funktionen mehr beeinträchtigt waren als die restlichen Teilnehmer. Auch dieser Faktor hätte das Gehen behindern können.

Vergleichbare Patientengruppen wichtig > Für eine unverfälschte Auswertung von Fragestellungen ist es also entscheidend, dass in Studien mehrere unabhängige Variablen für die Erklärung der abhängigen Variable herangezogen werden. Auf der anderen Seite sollten, wenn man eine univariate Analyse macht, die Patientengruppen vergleichbar sein, auch hinsichtlich ihrer Verlaufsprognose. Ist das nicht der Fall, steigt das Risiko einer möglicherweise rein zufälligen Anhäufung gleicher Faktoren.

Kommen als Auslöser für ein Symptom oder eine Krankheit also verschiedene Faktoren in Betracht, ist eine multivariate Auswertung in der Regel sinnvoller als eine univariate.

Prof. Dr. Jan Mehrholz

$\Rightarrow$ [1] Alexander LD et al. Association between gait asymmetry and brain lesion location in stroke patients. Stroke 2009; 40: $537-544$ 